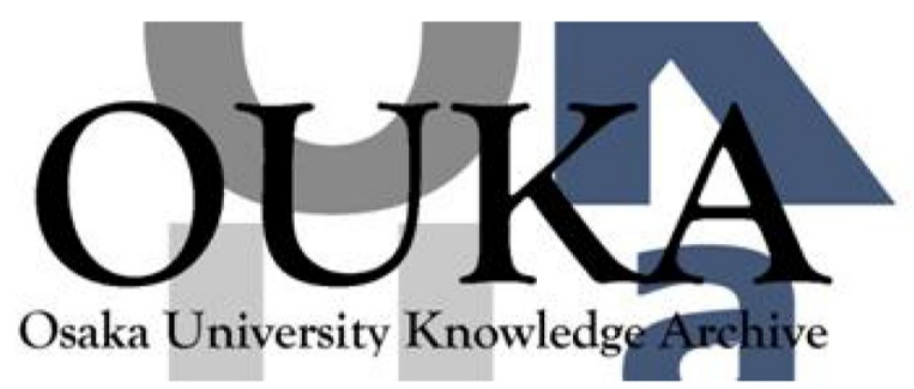

\begin{tabular}{|c|l|}
\hline Title & $\begin{array}{l}\text { Compact compound-eye projector using } \\
\text { superresolved projection }\end{array}$ \\
\hline Author(s) & Horisaki, Ryoichi; Tanida, Jun \\
\hline Citation & Optics Letters. 36(2) p. 121-p. 123 \\
\hline Issue Date & $2011-01-15$ \\
\hline oaire:version & VoR \\
\hline URL & https://hdl. handle. net/11094/2867 \\
\hline rights & \\
\hline Note & \\
\hline
\end{tabular}

Osaka University Knowledge Archive : OUKA

https://ir. Library. osaka-u. ac. jp/

Osaka University 


\title{
Compact compound-eye projector using superresolved projection
}

\author{
Ryoichi Horisaki* and Jun Tanida \\ Department of Information and Physical Sciences, Graduate School of Information Science and Technology, \\ Osaka University, 1-5 Yamadaoka, Suita, Osaka 565-0871, Japan \\ *Corresponding author: r.horisaki@ist.osaka-u.ac.jp
}

Received September 17, 2010; revised November 25, 2010; accepted November 26, 2010; posted December 2, 2010 (Doc. ID 135327); published January 5, 2011

We propose a compact compound-eye projector using superresolved projection. The optics are composed of multiple small suboptics (units) to reduce their thickness in the projector. Each of the suboptics adds a subpixel shift to a projected image for superresolved projection and has a color filter or a dispersive element for multicolor projection. The projected images in the units are determined via a model of the system in preprocessing. The images are projected by the suboptics and superimposed on a screen. In this Letter, we describe the system model and show the simulation results. (c) 2011 Optical Society of America

OCIS codes: $110.1758,100.6640$.

Projector hardware is drastically being reduced in size, owing to shrinkage of the optics used for projection and display devices [e.g., liquid-crystal displays (LCDs)]. A compact projector using compound-eye optics has been proposed [1]. The compound-eye optics are composed of multiple suboptics-each of which is referred to as a unit in this Letter. In the system, the projected images in the units are superimposed on a screen. When the number of units is $N_{u} \times N_{u}$, the thickness of the optics is $N_{u}$ times shorter than that of a conventional singleeye projector with the same field of view. However, the system requires a high-resolution or large display device, especially for multicolor projection, because the resolution of the superimposed image is the same as that of the projected image in each unit. This Letter solves this problem by employing superresolved projection, which has been proposed in a multiprojector system [2].

In the field of compact compound-eye cameras [3], some methods for spatially superresolved multicolor image capturing have been proposed [4,5]. We can also apply such methods to compound-eye projectors to realize superresolved multicolor projection. Projectors are similar to cameras, but they involve different imaging processes [6]. The main contribution in this Letter is the derivation of an imaging model of the proposed compound-eye projector. The model is used to determine the projected images in the units.

The proposed projector is illustrated in Fig. 1. Each unit can be considered as a projector in the multiprojector system [2]. The lenslets are arranged with irregularity to add a subpixel shift to the projected images for superresolved projection [4]. To realize multicolor projection while keeping the hardware compact, a color filter or a dispersive element is located on each lenslet. The scheme is inspired by a compound-eye spectral imaging system using superresolution [5]. The proposed projector cannot achieve the resolution of conventional multicolor projectors, because the image on the screen is multicolor, whereas the LCD is monochrome. However, proper design of the color filters or the dispersive elements may alleviate the reduction in resolution for certain images regarding the number of color channels.
The projected images in the units are determined in order to equalize the superimposed image and an arbitrary image in preprocessing. The determined images are projected by the optics in units passing through the color filter or the dispersive element. The resultant images are then superimposed on the screen.

Here we present the proposed system model and the results of simulations. For simplicity, the lenslets are treated as ideal optics, the screen is assumed to be located in the depth of field, and a one-dimensional model is adopted. Derivation of a more realistic model or extension of the model to two dimensions can be readily achieved with small modifications.

The model of the system with a single color can be described by

$$
\mathcal{G}(x)=\frac{1}{M} \sum_{u} \mathcal{F}_{u}\left(\lfloor x / M+0.5\rfloor-\mathcal{S}_{u}\right),
$$

where $\mathcal{G}$ and $\mathcal{F}_{u}$ are a superimposed image $\in \mathbb{R}^{N_{x} \times 1}$ and a projected image $\in \mathbb{R}^{N_{x^{\prime}} \times 1}$ in the $u$ th unit, respectively; $\mathbb{R}^{a \times b}$ denotes an $a \times b$ matrix of real numbers; and $\mathcal{S}_{u}$ is a shift caused by the position of the lenslet and parallax in the $u$ th unit. For simplicity, the shift is assumed to be an integer. $\lfloor\cdot\rfloor$ and $M$ are the floor function and the upsampling factor, which is calculated from $N_{x} / N_{x^{\prime}}$. The upsampling operation increases the sampling rate. This operation and $\mathcal{S}_{u}$ express a subpixel shift on the projected image [6].

The model of the system with multiple colors can be described by

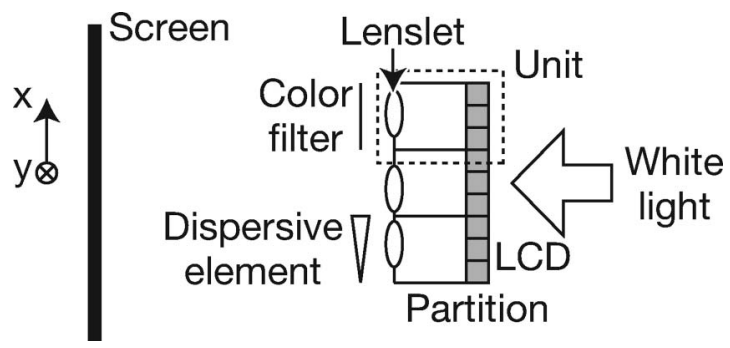

Fig. 1. Diagram of the proposed projector. 


$$
\mathcal{G}_{m}(x)=\frac{1}{M} \sum_{u} \mathcal{F}_{u}\left(\lfloor x / M+0.5\rfloor-\mathcal{S}_{m, u}\right) \mathcal{W}_{m, u},
$$

where $m$ is the index of the colors; $\mathcal{S}_{m, u}$ is a shift caused by a dispersive element for the $m$ th color, the position of the lenslet, and parallax in the $u$ th unit; and $\mathcal{W}_{m, u}$ is a weight of a color filter for the $m$ th color in the $u$ th unit.

As indicated by Eq. (2), a projected image of the $u$ th unit is upsampled with the factor $M$. The upsampled image is shifted and weighted by $\mathcal{S}_{m, u}$ and $\mathcal{W}_{m, u}$, respectively. The resultant images in all of the units are summed. For simplicity, $M=N_{u}=N_{x} / N_{x^{\prime}}$ is assumed.

The upsampling matrix $\boldsymbol{U} \in \mathbb{R}^{N_{x} \times N_{x^{\prime}}}$ can be defined by

$$
\boldsymbol{U}=\left[\begin{array}{cccc}
\mathbf{1} & \mathbf{0} & \ldots & \mathbf{0} \\
\mathbf{0} & \mathbf{1} & \ldots & \mathbf{0} \\
\vdots & \vdots & \ddots & \vdots \\
\mathbf{0} & \mathbf{0} & \ldots & \mathbf{1}
\end{array}\right]
$$

where $\mathbf{1}$ and $\mathbf{0}$ denote an $N_{u} \times 1$ vector whose elements are all 1 and an $N_{u} \times 1$ vector whose elements are all 0 , respectively.

$\boldsymbol{C}_{m, u}^{\prime} \in \mathbb{R}^{N_{x} \times N_{x}}$ denotes the shifting and the weighting operation for the $m$ th color in the $u$ th unit, which is written as

$$
\boldsymbol{C}_{m, u}^{\prime}(p, q)=\left\{\begin{array}{cc}
\mathcal{W}_{m, u} / M & \left(p=q+\mathcal{S}_{m, u}\right), \\
0 & \left(p \neq q+\mathcal{S}_{m, u}\right),
\end{array}\right.
$$

where $\boldsymbol{C}_{m, u}^{\prime}(p, q)$ is the $(p, q)$ th element in the matrix $\boldsymbol{C}_{m, u}^{\prime}$. The effects of practical defocus and aberrations in each of the lenslets can be included by use of $\boldsymbol{A}_{m, u} \boldsymbol{C}_{m, u}^{\prime}$ instead of $\boldsymbol{C}_{m, u}^{\prime}$, where $\boldsymbol{A}_{m, u} \in \mathbb{R}^{N_{x} \times N_{x}}$ is a matrix expressing the effects. For simplicity, $\boldsymbol{A}_{m, u}$ is assumed to be an identity matrix.

$C_{u} \in \mathbb{R}^{\left(N_{x} \times N_{m}\right) \times N_{x}}$ represents the shifting and the weighting operation for all of the colors in the $u$ th unit:

$$
\boldsymbol{C}_{u}=\left[\begin{array}{c}
\boldsymbol{C}_{0, u}^{\prime} \\
\boldsymbol{C}_{1, u}^{\prime} \\
\vdots \\
\boldsymbol{C}_{N_{m}-1, u}^{\prime}
\end{array}\right],
$$

where $N_{m}$ is the number of colors.

The projected image in the $u$ th unit is upsampled by $\boldsymbol{U}$, and the upsampled image is shifted and weighted by $\boldsymbol{C}_{u}$. Finally, the resultant images in all of the units are summed. The system matrix $\boldsymbol{H} \in \mathbb{R}^{\left(N_{x} \times N_{m}\right) \times\left(N_{x^{\prime}} \times N_{u}\right)}$ can be written as

$$
\begin{aligned}
& \boldsymbol{g}=\boldsymbol{H} \boldsymbol{f}=\left[\begin{array}{llll}
\boldsymbol{C}_{0} \boldsymbol{U} & \boldsymbol{C}_{1} \boldsymbol{U} & \cdots & \boldsymbol{C}_{N_{u}-1} \boldsymbol{U}
\end{array}\right] \boldsymbol{f} \\
& =\left[\begin{array}{cccc}
\boldsymbol{C}_{0,0}^{\prime} \boldsymbol{U} & \boldsymbol{C}_{0,1}^{\prime} \boldsymbol{U} & \cdots & \boldsymbol{C}_{0, N_{u}-1}^{\prime} \boldsymbol{U} \\
\boldsymbol{C}_{1,0}^{\prime} \boldsymbol{U} & \boldsymbol{C}_{1,1}^{\prime} \boldsymbol{U} & \cdots & \boldsymbol{C}_{1, N_{u}-1}^{\prime} \boldsymbol{U} \\
\vdots & \vdots & \ddots & \vdots \\
\boldsymbol{C}_{N_{m}-1,0}^{\prime} \boldsymbol{U} & \boldsymbol{C}_{N_{m}-1,1}^{\prime} \boldsymbol{U} & \cdots & \boldsymbol{C}_{N_{m}-1, N_{u}-1}^{\prime} \boldsymbol{U}
\end{array}\right] \boldsymbol{f},
\end{aligned}
$$

where $\boldsymbol{g}$ and $\boldsymbol{f}$ are a vectorized superimposed image $\in \mathbb{R}^{\left(N_{x} \times N_{m}\right) \times 1}$ and vectorized projected images $\in \mathbb{R}^{\left(N_{x^{\prime}} \times N_{u}\right) \times 1}$, respectively.

\begin{tabular}{|c|c|c|}
\hline$R$ & $G$ & $B$ \\
\hline$R$ & $G$ & $B$ \\
\hline$R$ & $G$ & $B$ \\
\hline
\end{tabular}

(a)

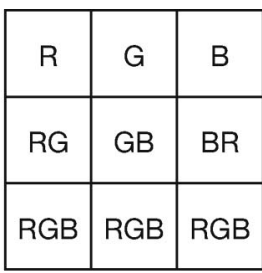

(b)

\begin{tabular}{|l|l|l|}
\hline$-2,-2$ & $0,-2$ & $+2,-2$ \\
\hline$-2,0$ & 0,0 & $+2,0$ \\
\hline$-2,+2$ & $0,+2$ & $+2,+2$ \\
\hline
\end{tabular}

(c)
Fig. 2. Setups of color filters in (a) system i and (b) system ii and setup of dispersive elements in (c) system iii.

Determination of the images projected by all of the units can be expressed as the following problem:

$$
\begin{aligned}
\hat{\boldsymbol{f}}= & \underset{\boldsymbol{f}}{\arg \min }\|\boldsymbol{g}-\boldsymbol{H} \boldsymbol{f}\|^{2} \\
& \text { subject to } 0 \leq \hat{\boldsymbol{f}}(p) \leq c, \quad \forall p,
\end{aligned}
$$

where $\hat{\boldsymbol{f}}(p)$ and $c$ are the $p$ th element of $\hat{\boldsymbol{f}}$ and the maximum value of a pixel in the projector [2], or

$$
\hat{\boldsymbol{f}} \underset{\boldsymbol{f}}{\arg \max } \mathcal{L}(\boldsymbol{f} \mid \boldsymbol{g}) \quad \text { subject to } \quad 0 \leq \hat{\boldsymbol{f}}(p) \leq c, \quad \forall p,
$$

where $\mathcal{L}(\cdot)$ is the likelihood function. Note that the number of elements in $\boldsymbol{g}\left(N_{x} \times N_{m}\right)$ is larger than that in $\boldsymbol{f}$ $\left(N_{x^{\prime}} \times N_{u}=N_{x}\right)$. Therefore, $\boldsymbol{g}$ cannot be perfectly identical to $\boldsymbol{H} \hat{f}$. In this Letter, Eq. ()ㅡㄹ is chosen. The problem in Eq. (8) can be solved based on the Richardson and Lucy method [7]. After solving the problem, $\hat{f}$ is displayed on the LCD.

In the simulations, the colors of the objects were red, green, and blue; therefore, $N_{m}=3$. The sizes of the original images were $129 \times 129$. The number of lenslets was set as $3 \times 3$. Three setups (i, ii, and iii) of the proposed system were simulated. Systems $\mathrm{i}$ and ii use single and multiple bandpass filters, as shown in Figs. 2(a) and 2(b), respectively. In the figures, $\mathrm{R}, \mathrm{G}$, and $\mathrm{B}$ represent $\overline{\text { red, }}$

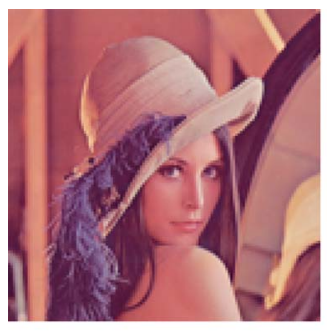

(a)

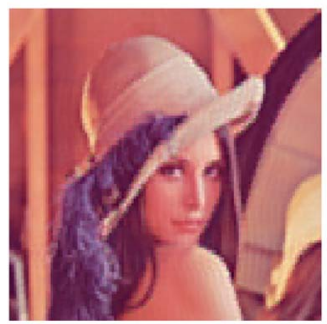

(c)

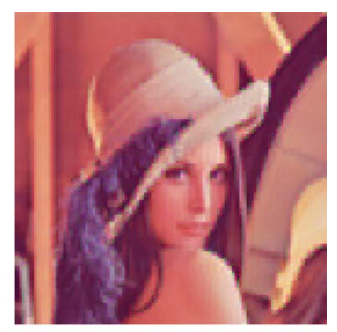

(b)

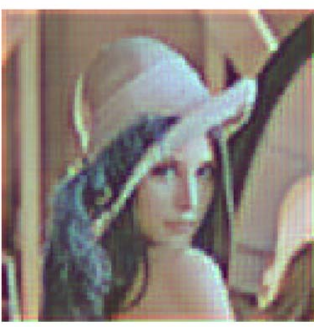

(d)
Fig. 3. (Color online) Simulation results with Lena: (a) original image and superimposed images obtained by (b) system i, (c) system ii, and (d) system iii. 


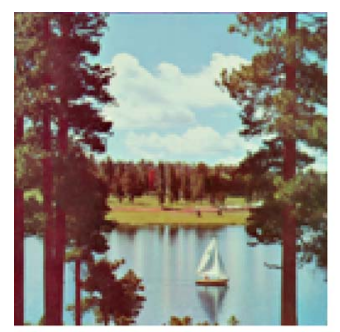

(a)

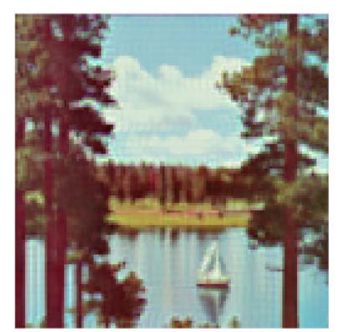

(c)

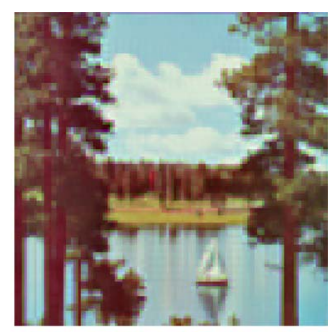

(b)

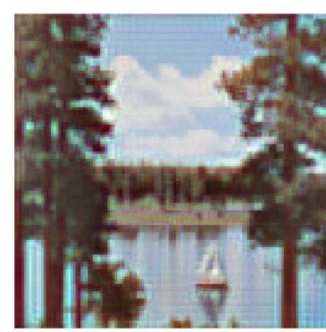

(d)
Fig. 4. (Color online) Simulation results with Lake: (a) original image and superimposed images obtained by (b) system i, (c) system ii, and (d) system iii.

green, and blue and indicate the passbands. In systems i and ii, $\mathcal{S}_{m, u}=\mathcal{R}_{u}$, where $\mathcal{R}_{u}$ is a random integer whose range is $[-1,+1]$. System iii uses dispersive elements. Here, $\mathcal{W}_{m, u}=1$ and $\mathcal{S}_{m, u}=\mathcal{A}_{u} m+\mathcal{R}_{u}$, where $\mathcal{A}_{u}$ is set as shown in Fig. 2(c). The figure shows $\mathcal{A}_{u}$ along the $x$ and $y$ axes. The value of $c$ in Eq. ( $\underline{8})$ was set to 9 .

Simulations with three images are shown in Figs. $\underline{3}-\underline{5}$. The original images are referred to as Lena, Lake, and RGB, respectively. Lena and Lake are natural images. The RGB image is composed of multiple characters in single colors.

Table 1 shows the peak signal-to-noise ratios (PSNRs) between the original images and the superimposed images in the three systems. As indicated in the table, system i was effective for objects with single colors

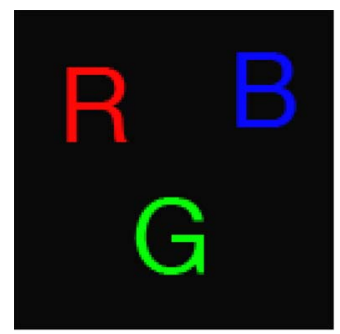

(a)

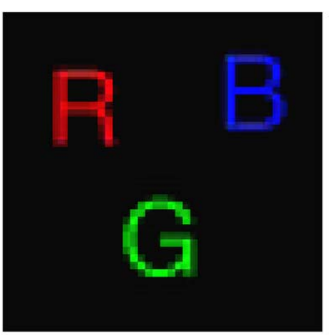

(c)

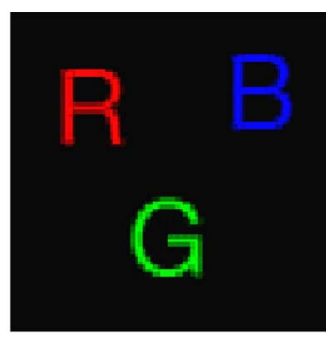

(b)

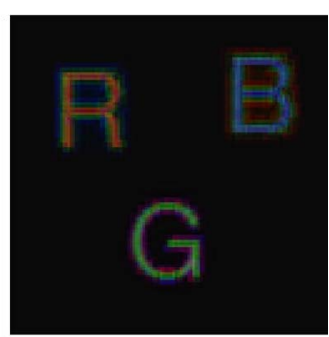

(d)
Fig. 5. (Color online) Simulation results with RGB: (a) original image and superimposed images obtained by (b) system i, (c) system ii, and (d) system iii.
Table 1. PSNRs (dB) between the Original Images and the Superimposed Images in Systems i, ii, and iii

\begin{tabular}{cccc}
\hline & System i & System ii & System iii \\
\hline Lena & 28.3 & 30.5 & 17.7 \\
Lake & 24.8 & 26.6 & 22.5 \\
RGB & 28.1 & 24.8 & 20.4 \\
\hline
\end{tabular}

and system ii was effective for objects with multiple colors. System iii was worse than the others. The results depended on the type of object.

The resolutions of the superimposed images for single colors in systems i and ii are roughly $B_{m} / N_{u}^{2}$ times lower than that of conventional projectors, where $B_{m}$ is the number of single bandpass filters of the $m$ th color, because units of multiple bandpass filters cannot be used. Estimation of the resolutions in the other cases requires further study. To maximize the resolutions in the proposed projector, the lens arrangement can be optimized by a method previously presented for compound-eye cameras [4].

The advantage of the proposed projector is its compactness. Increasing the number of units is one way to enhance this advantage. However, this increases the condition number in Eq. (6), which may degrade the superimposed image and require more iterations to determine the projected images.

We proposed a compact compound-eye projector using superresolved projection. In the system, the lenslets add subpixel shifts for superresolved projection, and color filters and dispersive elements are located in front of the units for multicolor projection. The projected images in the units are determined using a model of the system in preprocessing. The images are projected with the optical elements, and the resultant images are superimposed on the screen. The system model and three setups of the system were described. The system using a single bandpass filter was effective for objects with single colors, and the systems using single and multiple bandpass filters were effective for objects with multiple colors, for example, natural images.

\section{References}

1. M. Sieler, P. Schreiber, P. Dannberg, and A. Bräuer, Proc. SPIE 7716, 77161A (2010).

2. N. Damera-Venkata and N. L. Chang, in Proceedings of IEEE Conference on Computer Vision and Pattern Recognition (CVPR 'O') (IEEE, 2007), pp. 1-8.

3. J. Tanida, T. Kumagai, K. Yamada, S. Miyatake, K. Ishida, T. Morimoto, N. Kondou, D. Miyazaki, and Y. Ichioka, Appl. Opt. 40, 1806 (2001).

4. R. Horisaki, K. Kagawa, Y. Nakao, T. Toyoda, Y. Masaki, and J. Tanida, Appl. Phys. Express 3, 022501 (2010).

5. R. Horisaki, K. Choi, J. Hahn, J. Tanida, and D. J. Brady, Opt. Express 18, 19367 (2010).

6. N. Damera-Venkata and N. L. Chang, in Proceedings of the IEEE International Conference on Image Processing (ICIP, 2007), Vol. V, pp. 373-376.

7. W. H. Richardson, J. Opt. Soc. Am. 62, 55 (1972). 